Original article

\title{
Handgrip strength is an independent predictor of all-cause mortality in maintenance dialysis patients
}

\author{
Barbara Perez Vogt, Mariana Clementoni Costa Borges, Cassiana Regina de Goés, \\ Jacqueline Costa Teixeira Caramori* \\ Faculdade de Medicina de Botucatu, UNESP Univ Estadual Paulista, Department of Clinical Medicine, Botucatu, São Paulo, Brazil
}

\section{A R T I C L E I N F O}

\section{Article history:}

Received 20 November 2015

Accepted 24 March 2016

\section{Keywords:}

Dialysis

Handgrip strength

Mortality

Nutritional assessment

\begin{abstract}
S U M M A R Y
Background \& aims: Muscle wasting is associated with mortality in dialysis patients. The measurement of muscle mass has some limitations, while muscle strength assessment is simple, safe and allows the recognition of patients at risk of progressing to poor outcomes related to malnutrition. The aim of this study is verify if handgrip strength (HGS) is associated with all-cause mortality in patients in maintenance haemodialysis (HD) and peritoneal dialysis (PD).

Methods: This was an observational retrospective cohort study which included all patients in maintenance HD and PD from July 2012 to October 2014. Patients were followed-up until June 2015.

Results: Two-hundred sixty five patients were enrolled (218 HD and 47 PD) and they were followed for $13.4 \pm 7.9$ months. During the follow-up period, 53 patients (20\%) have died, 36 patients (13.6\%) have undergone renal transplantation, 13 patients (4.9\%) have switched off dialysis method and 5 patients (1.9\%) have transferred to another facility. The cut-off of HGS able to predict mortality was $22.5 \mathrm{~kg}$ for men and $7 \mathrm{~kg}$ for women. Using this cut-off to fit the Kaplan-Meier survival curve, the association of HGS with all-cause mortality for both genders was confirmed. Finally, in the multivariate analysis adjusted for demographic, clinical and nutritional variables, HGS remained significant predictor of mortality, independent of dialysis modality.

Conclusions: HGS cut-offs that predict mortality were $22.5 \mathrm{~kg}$ for men and $7 \mathrm{~kg}$ for women. HGS was associated with mortality independent of dialysis modality.
\end{abstract}

(C) 2016 Elsevier Ltd and European Society for Clinical Nutrition and Metabolism. All rights reserved.

\section{Introduction}

End-stage renal disease (ESRD) patients are at increased risk of protein energy wasting (PEW), which is characterized by muscle mass loss associated with inflammation [1]. Muscle mass wasting is highly prevalent among maintenance dialysis patients. Evidence of wasting can be noted in $18-75 \%$ of these patients, and it is an important predictor of morbidity and mortality [1]. Therefore,

Abbreviations: ESRD, end-stage renal disease; PEW, protein energy wasting; HGS, handgrip strength; CKD, chronic kidney disease; HD, haemodialysis; PD, peritoneal dialysis; CRP, C - reactive protein; BMI, body mass index; MAMC, midarm muscle circumference.

* Corresponding author. Faculdade de Medicina de Botucatu, UNESP, Univ Estadual Paulista Distrito de Rubião Junior, s/n 18618970, Botucatu, São Paulo, Brazil. Tel.: +55 143880 1171; fax: +55 1438822238

E-mail address: jteixeir@fmb.unesp.br (J.C.T. Caramori). markers of muscle mass and muscle function could be important predictors of outcomes in this population [2-4].

Several methods have been used for determining muscle mass in dialysis patients, i.e., dual-energy X-ray absorptiometry, bioelectrical impedance analysis, and anthropometry. However, all of these methods have some relevant disadvantages [5,6]. In this context, handgrip strength (HGS) has gained considerable attention as an indicator of nutrition status and muscle function in recent research. HGS is a validated method to assess nutritional status [7]. It is fast, safe, simple, reliable, non-invasive, painless, radiation-free, and low cost [8]. It shows high inter-rater reliability and may be useful as an early indication of malnutrition [9]. Moreover, it seems not affected by hydration status $[8,10]$, as the other methods for muscle mass assessment.

Studies with chronic kidney disease (CKD) patients have shown HGS is associated with malnutrition, assessed by Malnutrition Inflammation Score (MIS) [11] and renal outcomes (pre-dialysis mortality or reaching ESRD) [12]. In patients on maintenance 
dialysis, HGS is associated with malnutrition evaluated by Subjective Global Assessment $[13,14]$ and mortality $[4,10]$.

In view of the simplicity and advantages of HGS assessment in evaluating nutritional status and the significant influence of muscle wasting on mortality of patients on dialysis, the aims of this study are: to determine a HGS cut-off for men and women and verify its association with mortality in patients on maintenance dialysis.

\section{Materials and methods}

\subsection{Study design and subjects}

It was a retrospective cohort study which included prevalent haemodialysis (HD) and peritoneal dialysis (PD) patients for at least three months, treated at Hospital of Botucatu Medical School from July 2012 to August 2014. Patients younger than 18 years and those with missing nutritional assessment data were excluded. Patients were evaluated and followed until death, transplantation, switch off dialysis method, transfer to another facility or the end of follow-up in June 2015. The study protocol was approved by local research ethics committee.

Following demographic, clinical and laboratory data were obtained from medical records: gender, age, dialysis vintage, main cause of ESRD, diabetes, serum urea, creatinine, albumin and C reactive protein (CRP).

\subsection{Assessment of nutritional status and muscle function}

Anthropometric measurements were obtained from nutritional assessment performed after HD session or during routine outpatient care for PD patients. Body weight, height, mid-arm circumference and triceps skinfold thickness were measured. From these measurements, it was calculated body mass index (BMI), mid-arm muscle circumference (MAMC) and percent standard of MAMC was obtained from the National Health and Nutrition Examination Survey percentile distribution tables $[15,16]$.

HGS was measured during nutritional assessment, using Jamar ${ }^{\circledR}$ mechanical dynamometer with a precision of $1 \mathrm{~kg}$, in the dominant hand or in the non-fistula hand if implanted. Patients were instructed to self-adjust the dynamometer so that they fit comfortably to their hand size to get the best performance and to hold the grip with maximum force in response to a voice command, with the arm extended sideways from the body with the dynamometer facing away from the body. Three measurements were performed with intervals of about $30 \mathrm{~s}$ between each run and the maximum value was considered.

\subsection{Statistical analyses}

Data were expressed as mean \pm standard deviation or median (first and third quartiles), and frequencies were expressed as percentage. Comparisons between genders and between survival and non-survival patients were performed using $t$ Student's test or Mann Whitney. Frequencies were compared by qui-square test. Sensitivity and specificity analysis was performed to verify the best HGS cut-off able to predict mortality for men and women. A Receiver Operating Characteristic Curve Analysis (ROC curve) was constructed for each gender to verify the area under the curve and the significance of HGS on mortality prediction. Kaplan-Meier survival curves were fitted, and the difference between the curves was assessed by log-rank test. Cox proportional hazards analysis was used to assess independent predictors of mortality through models adjusted for variables significantly different between survival and non-survival patients. In this analysis, HG was included as a categorical variable according to the cut-off for each gender.
Statistical significance was accepted as a p-value $<0.05$. Statistical analysis was performed using SPSS 22.0.

\section{Results}

\subsection{Baseline characteristics}

This study enrolled 265 patients on maintenance dialysis, most of them on $\mathrm{HD}$ (82.3\%). Patients' age ranged from 18 to 91 years, and $54 \%$ were male. Diabetic nephropathy was the main cause of ESRD (31.1\%), followed by hypertensive nephrosclerosis (18.1\%). Patients mean BMI was $26.3 \pm 7.1 \mathrm{~kg} / \mathrm{m}^{2}$ and mean HGS was $18.7 \pm 11.2 \mathrm{~kg}$. Mean HGS for men was $24 \pm 11.6 \mathrm{~kg}$ and for women was $12.5 \pm 6.7 \mathrm{~kg}(\mathrm{p}<0.001)$.

Demographic, clinical and nutritional data of entire cohort and according to gender is presented in Table 1 .

Comparing patients according to dialysis methods, HD patients were majority male, with longer time of dialysis vintage and longer follow-up, and higher values of serum urea, creatinine and albumin $(p<0.05)$ than PD patients. HGS and frequency of outcomes were not different between HD and PD.

\subsection{Comparisons between survival and non-survivals patients}

Age, prevalence of diabetes, dialysis vintage and CRP were higher among non-survival patients, while serum urea, creatinine and albumin were higher among survival patients (Table 2).

\subsection{Follow-up}

Patients were followed-up for a mean $13.4 \pm 7.9$ months (minimum 0.6 and maximum 30.9 months). During this period, 53 patients (20\%) have died, 36 patients $(13.6 \%)$ have received renal transplantation, 13 patients $(4.9 \%)$ have switched off dialysis method and 5 patients (1.9\%) have been transferred to another facility.

\subsection{ROC curve}

According to ROC curves, HGS cut-off able to predict mortality were $22.5 \mathrm{~kg}$ for male, with $61 \%$ sensitivity and $76 \%$ specificity (AUC 0.689; CI 95\% 0.575-0.803; p < 0.003) and $7 \mathrm{~kg}$ for female, with 83\% sensitivity and 35.7\% specificity (AUC 0.615; CI 95\% 0.493-0.737; $\mathrm{p}=0.06$ ) (see Fig. 1).

\subsection{Kaplan-Meier survival analysis}

Survival probability analysis (Kaplan-Meier) confirmed the association of HGS with all-cause mortality for both gender (Fig. 2). The results showed statistical significance for both male (Fig. 2A) $(\mathrm{p}=0.003)$ and female (Fig. $2 \mathrm{~B})(\mathrm{p}=0.004)$.

\subsection{Cox proportional hazards analysis}

Models were fitted using cox proportional hazards analysis with HGS as a categorical variable, considering the cut-off for each gender (Table 3). First, crude analysis showed HGS as a significant predictor of mortality. HGS remained significant predictor of mortality even after adjustments.

\section{Discussion}

The present study showed HGS was able to identify increased risk of all-cause mortality in dialysis patients, with different cutoffs for genders. HGS is a measure widely used in clinical 
Table 1

Baseline characteristics of 265 included patients and comparison between males and females.

\begin{tabular}{|c|c|c|c|c|}
\hline Characteristic & $\begin{array}{l}\text { Total } \\
(\mathrm{n}=265)\end{array}$ & $\begin{array}{l}\text { Men } \\
(n=143)\end{array}$ & $\begin{array}{l}\text { Women } \\
(\mathrm{n}=122)\end{array}$ & $\mathrm{P}$ \\
\hline Age (years) & $58 \pm 15.2$ & $59.2 \pm 14.8$ & $57.1 \pm 14.5$ & 0.7 \\
\hline Dialysis method [HD(\%)/PD(\%)] & $218(82.3) / 47(17.7)$ & $124(86.7) / 19(13.3)$ & $94(77) / 28(23)$ & 0.04 \\
\hline Diabetes $[\mathrm{n}(\%)]$ & $122(46.2)$ & $62(43.4)$ & $60(49.6)$ & 0.31 \\
\hline Follow-up (months) & $13.4 \pm 7.9$ & $13.8 \pm 7.9$ & $14.6 \pm 8.3$ & 0.88 \\
\hline Dialysis vintage (months) & $14.3(2.8 ; 36.7)$ & $15.4(3.9 ; 42.6)$ & $19.4(6.9 ; 40.6)$ & 0.58 \\
\hline \multicolumn{5}{|c|}{ Cause of end-stage renal disease [n(\%)] } \\
\hline Diabetic nephropathy & $83(31.3)$ & $46(32.2)$ & $37(30.3)$ & \multirow[t]{6}{*}{0.49} \\
\hline Hypertensive nephrosclerosis & $48(18.1)$ & $25(17.5)$ & $23(18.9)$ & \\
\hline Unknown & $47(17.7)$ & $23(16.1)$ & $24(19.7)$ & \\
\hline Chronic glomerulonephritis & $26(9.8)$ & $14(9.8)$ & $12(9.8)$ & \\
\hline ADPKD & $8(3.0)$ & $7(4.9)$ & $1(0.8)$ & \\
\hline Other & $53(20.0)$ & $28(19.6)$ & $25(20.5)$ & \\
\hline $\operatorname{BMI}\left(\mathrm{kg} / \mathrm{m}^{2}\right)$ & $26.3 \pm 7.1$ & $25.3 \pm 5.0$ & $26.5 \pm 6.2$ & 0.02 \\
\hline Percent standard of MAMC (\%) & $99.7 \pm 16.8$ & $91.8 \pm 11.4$ & $109.3 \pm 18.0$ & $<0.01$ \\
\hline Handgrip strength (kg) & $18.7 \pm 11.2$ & $24.0 \pm 11.4$ & $12.5 \pm 6.5$ & $<0.01$ \\
\hline Serum urea $(\mathrm{mg} / \mathrm{dl})$ & $104.6 \pm 34$ & $111.1 \pm 34.6$ & $101.9 \pm 32.5$ & 0.01 \\
\hline Serum creatinine (mg/dl) & $8.5 \pm 3$ & $9.2 \pm 2.9$ & $8.1 \pm 2.7$ & $<0.01$ \\
\hline Serum albumin (g/dl) & $3.8 \pm 0.5$ & $3.9 \pm 0.5$ & $3.8 \pm 0.6$ & 0.22 \\
\hline$C$ reactive protein $(\mathrm{mg} / \mathrm{dl})$ & $1(0.5-1.9)$ & $1.1(0.5 ; 1.9)$ & $0.9(0.5 ; 1.8)$ & 0.97 \\
\hline Haemoglobin (g/dl) & $11.1 \pm 1.8$ & $11.57 \pm 1.8$ & $11.21 \pm 1.6$ & 0.10 \\
\hline Serum bicarbonate (mEq/L) & $22.4 \pm 2.8$ & $22.39 \pm 2.8$ & $22.53 \pm 3.0$ & 0.70 \\
\hline $\mathrm{Kt} / \mathrm{V}$ & $1.5 \pm 0.4$ & $1.4 \pm 0.3$ & $1.7 \pm 0.5$ & $<0.01$ \\
\hline
\end{tabular}

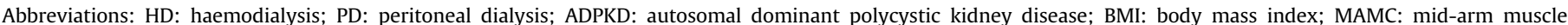
circumference.

Percent standard of MAMC was obtained from the National Health and Nutrition Examination Survey percentile distribution tables [15].

practice, due to its ability to easily suggest protein energy malnutrition. Moreover, it is simple, fast, non-invasive, and represents a reliable malnutrition marker [8,9]. Malnutrition has been independently associated with poor outcomes, such as hospitalization, longer length of hospital stay and mortality [17]. Studies have shown HGS as a good nutritional status indicator, able to diagnose early malnutrition due to changes in muscle function $[7-9,18]$.

There is a lack of consensus on HGS reference values for patients on dialysis, as well on the methodology to measure HGS. Leal et al. showed in a systematic review that dialysis patients HGS values range from 12 to $38 \mathrm{~kg}$ for men and from 12 to $26 \mathrm{~kg}$ for women
[19]. The mean HGS found in the present study meets this finding. It was $24 \mathrm{~kg}$ for men and $12.5 \mathrm{~kg}$ for women.

Matos et al. [4] showed values $28.3 \mathrm{~kg}$ for men and $21.5 \mathrm{~kg}$ for women were the best cut-offs to predict mortality in haemodialysis patients. In this study, the values were lower and more disparate: $22.5 \mathrm{~kg}$ for men and $7 \mathrm{~kg}$ for women. This result was expected, because gender is already well known as an important determinant of HGS [20].

As well as gender, age is another factor that influences muscle strength [20-22]. Uraemic phenotype is known to be characterized by premature ageing, which accelerates characteristics as

Table 2

Comparison between demographic, clinical and nutritional characteristics of survivals and non-survivals patients.

\begin{tabular}{|c|c|c|c|}
\hline Characteristic & Survival $(\mathrm{n}=212)$ & Non-survival $(\mathrm{n}=53)$ & $\mathrm{P}$ \\
\hline Age (years) & $57 \pm 15.5$ & $62.9 \pm 13$ & $<0.01$ \\
\hline Gender [Male(\%)] & $118(55.7)$ & $25(47.2)$ & 0.27 \\
\hline Dialysis method (HD/PD) & $171(80.7) / 41(19.3)$ & $47(88.7) / 6(11.3)$ & 0.17 \\
\hline Diabetes $[\mathrm{n}(\%)]$ & $91(42.9)$ & $31(59.6)$ & 0.03 \\
\hline Follow-up (months) & $13.8 \pm 7.9$ & $11.7 \pm 7.5$ & 0.08 \\
\hline Dialysis vintage (months) & $11.8(2.4 ; 34.9)$ & $26.3(9.1 ; 56.6)$ & $<0.01$ \\
\hline \multicolumn{4}{|c|}{ Cause of end-stage renal disease $[n(\%)]$} \\
\hline Diabetic nephropathy & $63(29.7)$ & $20(37.7)$ & 0.65 \\
\hline Hypertensive nephrosclerosis & $37(17.5)$ & $11(20.8)$ & \\
\hline Unknown & $41(19.3)$ & $6(11.3)$ & \\
\hline Chronic glomerulonephritis & $22(10.4)$ & $4(7.5)$ & \\
\hline ADPKD & $7(3.3)$ & $1(1.9)$ & \\
\hline Other & $42(19.8)$ & $11(20.8)$ & \\
\hline BMI $\left(\mathrm{kg} / \mathrm{m}^{2}\right)$ & $26.3 \pm 7.1$ & $26.2 \pm 6.9$ & 0.94 \\
\hline Percent standard of MAMC (\%) & $99.6 \pm 16.3$ & $99.8 \pm 18.8$ & 0.96 \\
\hline Handgrip strength $(\mathrm{kg})$ & $19.9 \pm 11.3$ & $13.6 \pm 9.6$ & $<0.01$ \\
\hline Serum urea $(\mathrm{mg} / \mathrm{dl})$ & $106.6 \pm 34.2$ & $96.4 \pm 31.6$ & 0.05 \\
\hline Serum creatinine $(\mathrm{mg} / \mathrm{dl})$ & $8.7 \pm 3.1$ & $7.8 \pm 2.5$ & 0.05 \\
\hline Serum albumin $(\mathrm{g} / \mathrm{dl})$ & $3.8 \pm 0.5$ & $3.7 \pm 0.5$ & 0.06 \\
\hline$C$ reactive protein $(\mathrm{mg} / \mathrm{dl})$ & $0.9(0.5 ; 1.8)$ & $1.5(0.7 ; 2.2)$ & 0.01 \\
\hline Haemoglobin (g/dl) & $11.4 \pm 1.8$ & $11.2 \pm 1.5$ & 0.37 \\
\hline Serum bicarbonate $(\mathrm{mEq} / \mathrm{L})$ & $22.3 \pm 2.7$ & $22.9 \pm 3.5$ & 0.16 \\
\hline $\mathrm{Kt} / \mathrm{V}$ & $1.5 \pm 0.4$ & $1.5 \pm 0.4$ & 0.93 \\
\hline
\end{tabular}

Abbreviations: HD: haemodialysis; PD: peritoneal dialysis; ADPKD: autosomal dominant polycystic kidney disease; BMI: body mass index; MAMC: mid-arm muscle circumference. 

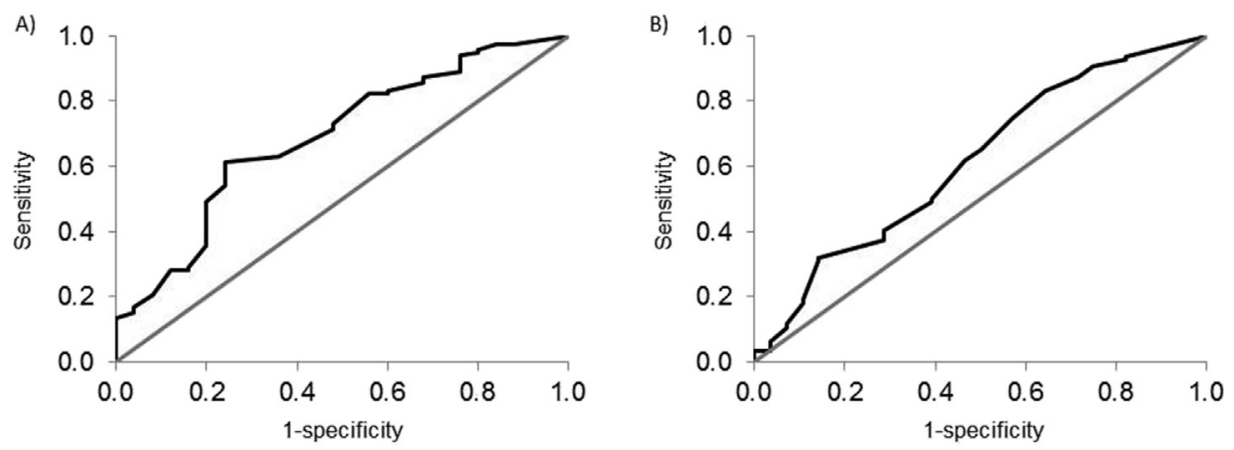

Fig. 1. ROC curves showing prediction of mortality by handgrip strength (A) for men and (B) for women.
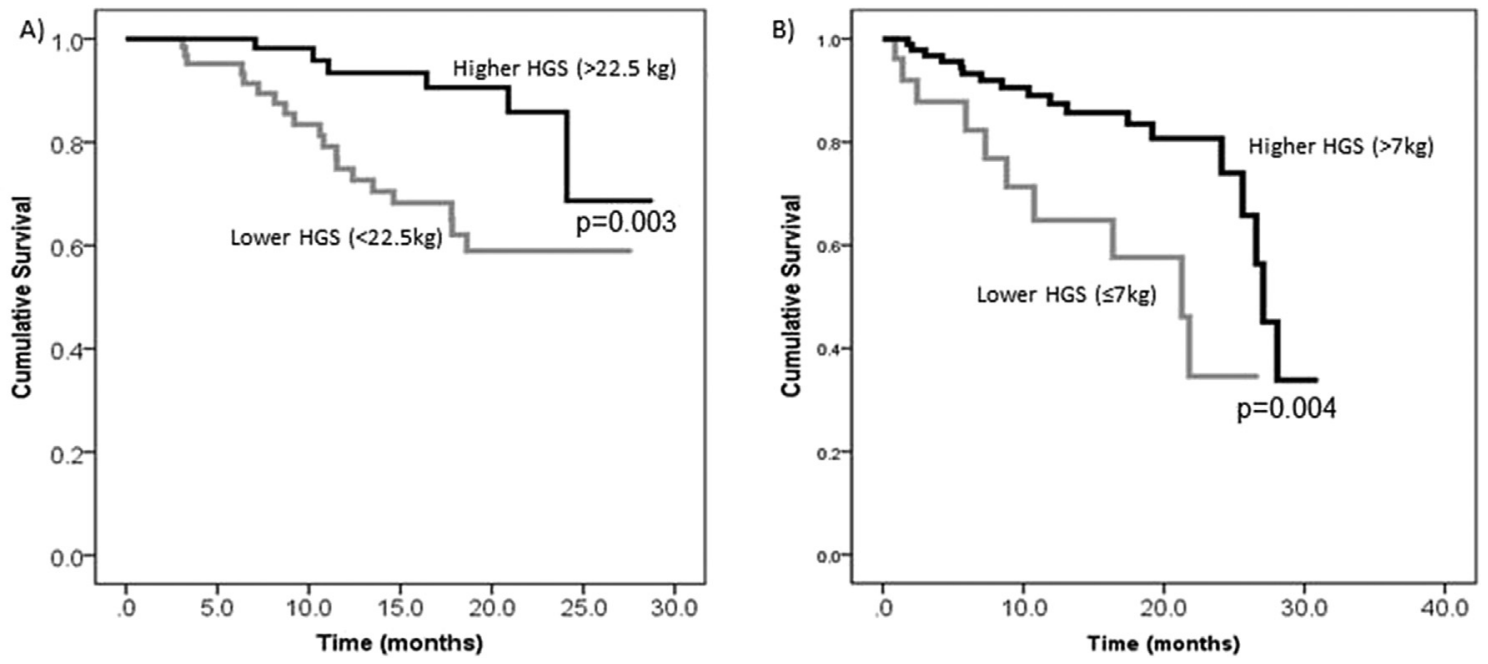

Fig. 2. Kaplan-Meier survival analysis of handgrip strength (A) for men and (B) for women.

osteoporosis, atherosclerosis, frailty and muscle wasting [23]. Therefore, there is a disconnection between calendar age and biological age in CKD that affects the muscle of these patients [24]. Nutritional status, which is usually reduced in chronic diseases, also exerts a substantial impact on muscle strength [7,12,25]. Other factors are associated with chronic diseases and contributes to muscle weakness as well: disease severity, comorbidities, medical treatment, and immobilisation [7].

Clinical and biochemical characteristics (age, diabetes, dialysis vintage, serum albumin, creatinine, urea and CRP) were different between survival and non-survival groups. These variables are associated with comorbidities, inflammation and nutritional status, therefore they can influence muscle strength. However, after

Table 3

Cox Proportional Hazard Analysis for evaluating the impact of handgrip strength on all-cause mortality in dialysis patients.

\begin{tabular}{llcc}
\hline & & HR $(95 \% \mathrm{CI})$ & $\mathrm{P}$ \\
\hline Crude & & $0.37(0.21-0.64)$ & $<0.01$ \\
Model 1 & Age & $1.01(0.99-1.03)$ & 0.46 \\
& Dialysis vintage & $1.01(0.99-1.01)$ & 0.12 \\
& Diabetes & $1.6(0.88-2.88)$ & 0.12 \\
& C reactive protein & $1.05(0.99-1.1)$ & 0.06 \\
& Serum creatinine & $0.88(0.78-0.99)$ & 0.047 \\
& Handgrip strength & $0.49(0.27-0.89)$ & 0.019 \\
\hline
\end{tabular}

Handgrip strength is a categorical variable according to the cut-off for each gender. multivariate analysis which included these variables, only HGS remains associated with mortality.

Muscle mass assessed by MAMC wasn't significantly different between survival and non-survival groups. On the other hand, muscle function assessed by HGS was statistically different between these groups. Isoyama et al. [3] showed decreased muscle strength was more strongly associated with mortality than decreased muscle mass in patients on dialysis. Therefore, muscle mass loss and muscle strength loss seems not to occur simultaneously.

Many studies have shown the prediction of low muscle mass in mortality in patients on dialysis [26-28]. As the decrease of muscle strength may occur before the decrease of muscle mass, it seems prudent to suggest that treatment options in dialysis patients should target not only maintenance or increase of muscle mass but also muscle functionality [3].

This study had some methodological limitations. Sample size was from a single centre, and longitudinal changes in HGS measurements were not assessed during follow-up. Because this is an observational study, there are confounding factors in the prediction of mortality. Despite these limitations, this study showed an association between HGS and mortality, independent of dialysis modality and other characteristics usually associated with poor outcomes.

In conclusion, HGS is an independent predictor of all-cause mortality in patients on maintenance dialysis. HGS cut-offs that predicted mortality were $22.5 \mathrm{~kg}$ for men and $7 \mathrm{~kg}$ for women. HGS 
was associated with mortality independent of dialysis modality. Moreover, measurement of HGS can be useful in clinical practice. It is noteworthy that the results of this study are significant and may base robust studies with longitudinal assessments to evaluate the influence of changes in muscle strength and mortality risk.

\section{Statement of authorship}

B. P. Vogt and M. C. C. Borges contributed to the conception and design of the research; B. P. Vogt, M. C. C. Borges C. R. Goes, and J. C. T. Caramori contributed to the acquisition, analysis, or interpretation of the data; B. P. Vogt and M. C. C. Borges drafted the manuscript; B. P. Vogt, M. C. C. Borges, C. R. Goes and J. C. T. Caramori critically revised the manuscript; and B. P. Vogt, M. C. C. Borges, C. R. Goes and J. C. T. Caramori agree to be fully accountable for ensuring the integrity and accuracy of the work. All authors read and approved the final manuscript.

\section{Conflict of interest}

The authors declare they have no conflicts of interest.

\section{Acknowledgements}

A master's degree scholarship was provided to MCCB, and a doctorate scholarship was provided to BPV by Coordination for the Improvement of Higher Education Personnel (Coordenação de Aperfeiçoamento de Pessoal de Nível Superior), an organization of the Brazilian federal government under the Ministry of Education.

\section{References}

[1] Fouque D, Kalantar-Zadeh K, Kopple J, Cano N, Chauveau P, Cuppari L, et al. A proposed nomenclature and diagnostic criteria for protein-energy wasting in acute and chronic kidney disease. Kidney Int 2008;73(4):391-8.

[2] Yoda M, Inaba M, Okuno S, Yoda K, Yamada S, Imanishi Y, et al. Poor muscle quality as a predictor of high mortality independent of diabetes in hemodialysis patients. Biomed Pharmacother 2012;66(4):266-70.

[3] Isoyama N, Qureshi AR, Avesani CM, Lindholm B, Bàràny P, Heimbürger $O$, et al. Comparative associations of muscle mass and muscle strength with mortality in dialysis patients. Clin J Am Soc Nephrol 2014 7;9(10):1720-8.

[4] Matos CM, Silva LF, Santana LD, Santos LS, Protásio BM, Rocha MT, et al Handgrip strength at baseline and mortality risk in a cohort of women and men on hemodialysis: a 4-year study. J Ren Nutr 2014:24(3):157-62.

[5] Avesani CM, Draibe SA, Kamimura MA, Cendoroglo M, Pedrosa A, Castro ML et al. Assessment of body composition by dual energy X-ray absorptiometry skinfold thickness and creatinine kinetics in chronic kidney disease patients. Nephrol Dial Transpl 2004;19(9):2289-95.

[6] Kamimura MA, Avesani CM, Cendoroglo M, Canziani MEF, Draibe SA, Cuppari L. Comparison of skinfold thicknesses and bioelectrical impedance analysis with dual-energy X-ray absorptiometry for the assessment of body fat in patients on long-term haemodialysis therapy. Nephrol Dial Transpl 2003;18(1):101-5.
[7] Norman K, Stobäus N, Gonzalez MC, Schulzke J-D, Pirlich M. Hand grip strength: outcome predictor and marker of nutritional status. Clin Nutr 2011;30(2):135-42.

[8] Leal VO, Stockler-Pinto MB, Farage NE, Aranha LN, Fouque D, Anjos LA, et al. Handgrip strength and its dialysis determinants in hemodialysis patients. Nutr 2011;27(11-12):1125-9.

[9] Flood A, Chung A, Parker H, Kearns V, O'Sullivan TA. The use of hand grip strength as a predictor of nutrition status in hospital patients. Clin Nutr 2014:33(1):106-14.

[10] Wang AY-M, Sea MM-M, Ho ZS-Y, Lui S-F, Li PK-T, Woo J. Evaluation of handgrip strength as a nutritional marker and prognostic indicator in peritoneal dialysis patients. Am J Clin Nutr 2005;81(1):79-86.

[11] Amparo FC, Cordeiro AC, Carrero JJ, Cuppari L, Lindholm B, Amodeo C, et al. Malnutrition-inflammation score is associated with handgrip strength in nondialysis-dependent chronic kidney disease patients. J Ren Nutr 2013;23(4):283-7.

[12] Chang Y-T, Wu H-L, Guo H-R, Cheng Y-Y, Tseng C-C, Wang M-C, et al Handgrip strength is an independent predictor of renal outcomes in patients with chronic kidney diseases. Nephrol Dial Transpl 2011;26(11):3588-95.

[13] Qureshi AR, Alvestrand A, Danielsson A, Divino-Filho JC, Gutierrez A Lindholm B, et al. Factors predicting malnutrition in hemodialysis patients: a cross-sectional study. Kidney Int 1998;53(3):773-82.

[14] Carrero JJ, Chmielewski M, Axelsson J, Snaedal S, Heimbürger O, Bárány P, et al. Muscle atrophy, inflammation and clinical outcome in incident and prevalent dialysis patients. Clin Nutr 2008;27(4):557-64.

[15] Frisancho AR. New norms of upper limb fat and muscle areas for assessment of nutritional status. Am J Clin Nutr 1981;34(11):2540-5.

[16] Frisancho AR. Anthropometric standards for the assessment of growth and nutritional status. 1990.

[17] Correia MITD, Waitzberg DL. The impact of malnutrition on morbidity, mortality, length of hospital stay and costs evaluated through a multivariate model analysis. Clin Nutr 2003;22(3):235-9.

[18] Humphreys J, de la Maza P, Hirsch S, Barrera G, Gattas V, Bunout D. Muscle strength as a predictor of loss of functional status in hospitalized patients. Nutr 2002;18(7-8):616-20.

[19] Leal VO, Mafra D, Fouque D, Anjos LA. Use of handgrip strength in the assessment of the muscle function of chronic kidney disease patients on dialysis: a systematic review. Nephrol Dial Transpl 2011;26(4):1354-60.

[20] Budziareck MB, Pureza Duarte RR, Barbosa-Silva MCG. Reference values and determinants for handgrip strength in healthy subjects. Clin Nutr 2008;27(3): 357-62.

[21] Hughes VA, Frontera WR, Wood M, Evans WJ, Dallal GE, Roubenoff R, et al. Longitudinal muscle strength changes in older adults: influence of muscle mass, physical activity, and health. J Gerontol 2001;56(5):B209-17.

[22] Goodpaster BH, Park SW, Harris TB, Kritchevsky SB, Nevitt M, Schwartz AV, et al. The loss of skeletal muscle strength, mass, and quality in older adults: the health, aging and body composition study. J Gerontol 2006;61(10): 1059-64.

[23] Kooman JP, Kotanko P, Schols AMWJ, Shiels PG, Stenvinkel P. Chronic kidney disease and premature ageing. Nat Rev Nephrol 2014;10(12):732-42.

[24] Stenvinkel P. Larsson TE. Chronic kidney disease: a clinical model of premature aging. Am J Kidney Dis 2013;62(2):339-51.

[25] Mithal A, Bonjour J-P, Boonen S, Burckhardt P, Degens H, El Hajj Fuleihan G, et al. Impact of nutrition on muscle mass, strength, and performance in older adults. Osteoporos Int J 2013;24(5):1555-66.

[26] Noori N, Kopple JD, Kovesdy CP, Feroze U, Sim JJ, Murali SB, et al. Mid-arm muscle circumference and quality of life and survival in maintenance hemodialysis patients. Clin J Am Soc Nephrol 2010;5(12):2258-68.

[27] de Araújo IC, Kamimura MA, Draibe SA, Canziani MEF, Manfredi SR, Avesani CM, et al. Nutritional parameters and mortality in incident hemodialysis patients. J Ren Nutr 2006;16(1):27-35.

[28] Beddhu S, Pappas LM, Ramkumar N, Samore M. Effects of body size and body composition on survival in hemodialysis patients. J Am Soc Nephrol $2003 ; 14(9): 2366-72$. 